\title{
Étude des bactéries multirésistantes des effluents hospitaliers d'un centre hospitalier et universitaire (CHU) de la ville d'Abidjan (Côte d'Ivoire).
}

GUESSENND N.K ${ }^{1}$, OUATTARA M.B'1,2, OUATTARA N.D ${ }^{1}$, NEVRY R. K. ${ }^{2}$, GBONON V1'., TIEKOURA K. B ${ }^{1}$., DOSSO M. ${ }^{1}$ et le GER BMR ${ }^{3}$

1 : Institut Pasteur, Abidjan, Côte d'Ivoire

2 : Laboratoire de microbiologie et de biotechnologie, Université Nangui Abrogoua, Côte d'Ivoire

3 : GER BMR : Groupe d'Etude et de Recherche sur les bactéries multirésistantes

Auteur correspondant : guessennd@yahoo.fr

Original submitted in on 30 30 th 2013 Published online at www.m.elewa.org on 30th September 2013. https://dx.doi.org/10.4314/jab.v69i0.95071

\section{RÉSUMÉ}

Objectif : Les effluents non traitées générés par les activités hospitalières peuvent contribuer largement à la dissémination des bactéries multirésistantes (BMR) dans l'environnement. L'objectif était de détecter des Bactéries Multi-Résistantes (BMR) dans les effluents hospitaliers et d'évaluer leur niveau de résistance visà-vis des antibiotiques.

Méthodologie et résultats : Le dénombrement de la flore totale et de la flore résistante ont été effectués respectivement sur la gélose PCA et les géloses sélectives contenant de la ceftazidime (milieux maisons). La concentration moyenne des échantillons en flore totale était de 10,3.105 UFC/ml, inférieure à la flore totale généralement présente dans les eaux usées $\left(10^{8} \mathrm{UFC} / \mathrm{ml}\right)$. Les bactéries suivantes ont été les plus isolées E. coli, K. pneumoniae, $P$. aeruginosa, Staphylococcus aureus et $A$. baumanii. La plus part des souches étaient résistantes à trois familles d'antibiotiques (bêtalactamines, aminosides et fluoroquinolones).

Conclusion : II ressort de cette étude que les effluents du Centre Hospitalier et Universitaire (CHU) de Cocody (Abidjan, Côte d'Ivoire) renferme la plupart des bactéries multi-résistantes impliquées dans les infections nosocomiales à savoir les entérobactéries et Acinetobacter baumanii producteurs de BétaLactamines à Spectre Elargi (BLSE), Pseudomonas résistants à la ceftazidime (PARC), Staphylococcus aureus résistant à la méthicilline (SARM). Ces bactéries multirésistantes présentent une résistance à deux ou trois familles d'antibiotiques. Les bactéries multirésistantes présentes dans les effluents hospitaliers rejetés sans traitement préalable sont susceptibles de causer des problèmes de santé publique.

Mots clés : antibiotiques, bactéries multi-résistantes, effluents hospitaliers.

\section{Abstract}

Objective: Untreated effluents generated by hospital activities can contribute significantly to the spread of multiresistant bacteria (MRB) in the environment. The objective was to detect Multi-Resistant bacteria (MRB) in hospital effluents and assess their level of resistance to antibiotics

Methods and Results: The count of the total flora and resistant flora was made respectively on PCA agar and selective agar containing ceftazidime (media). The average concentration of total flora samples was 
$10.310^{5} \mathrm{CFU} / \mathrm{ml}$, less than the total flora normally present in wastewater $\left(10^{8} \mathrm{CFU} / \mathrm{ml}\right)$. The following bacteria were the most isolated $E$. coli, K. pneumoniae, $P$. aeruginosa, Staphylococcus aureus and $A$. baumanii. Most of the strains were resistant to three classes of antibiotics (beta-lactams, aminoglycosides and fluoroquinolones)

Conclusion: It appears from this study that the effluent of University of Cocody teaching hospital (Abidjan, Côte d'Ivoire) contains most of multi-resistant bacteria involved in nosocomials infections such as Acinetobacter baumanii and Enterobacteriaceae producing beta-lactam antibiotics in Expanded spectrum (ESBL) Pseudomonas resistant to ceftazidime (PARC), Staphylococcus aureus resistant to methicillin (MRSA). These resistant bacteria are resistant to two or three families of antibiotics. Multiresistant bacteria present in the hospital effluents discharged without treatment are likely to cause public health problems.

Keywords: hospital effluents, multi-resistant bacteria, antibiotics

\section{INTRODUCTION}

Les effluents générés par les activités hospitalières peuvent présenter un danger potentiel pour l'homme et son environnement. Cela, compte tenu de la nature et de l'importance des substances qu'ils contiennent comme les résidus médicamenteux, les réactifs chimiques, les antiseptiques, les détergents, les révélateurs et fixateurs de radiographies. En outre, les effluents hospitaliers contiennent des micro- bactéries potentiellement pathogènes, des virus et des champignons. Ces déchets liquides sont évacués au même titre que les rejets urbains classiques vers le réseau d'assainissement communal sans traitement préalable et surtout vers les eaux de surface comme les eaux lagunaires (Coralie et al., 2002). Les effluents non traitées générés par les activités hospitalières peuvent contribuer largement à la dissémination des bactéries multirésistantes (BMR) dans l'environnement. Les BMR des effluents proviennent essentiellement soit des produits biologiques (sang, urines, pus etc.) des patients colonisés (Akoua et al., 2004, Guessennd et al. 2008), soit de transfert horizontal de gènes

\section{MATÉRIEL ET MÉTHODES}

II s'agit d'une étude expérimentale sur la caractérisation des BMR dans les eaux usées non traitées du Centre Hospitalier Universitaire (CHU) de Cocody. Cette étude s'est déroulée du 1er septembre 2009 au 30 décembre 2009. Trente millilitres $(30 \mathrm{ml})$ d'eau ont été prélevés dans le regard principal collecteur des eaux usées de tous les services cliniques, des laboratoires, de la morgue, de la cuisine et de la buanderie du $\mathrm{CHU}$ de Cocody. La fréquence des prélèvements a été journalière à heures précises en fonction des activités de l'hôpital : le matin à 7 heures, l'après-midi à 13 heures et à 17 heures. Au total 15 prélèvements de résistance entre les souches multi-résistantes infectieuses et des souches environnementales (Thomas et al., 2007). Ces BMR généralement responsables des infections nosocomiales peuvent se retrouver dans l'environnement en particulier dans les eaux de surface (Senka et al., 2003; Mehmet et al., 2008) et être impliquées dans les infections communautaires (Theresa et al., 1984, Rhazi et al., 2007). En Côte d'Ivoire, la résistance des bactéries aux antibiotiques est devenu une véritable préoccupation pour le personnel soignant et pour les malades, comme l'atteste plusieurs publications (Dadié et al., 2003; Akoua et al., 2004, Guessennd et al., 2004; Gbonon et al., 2007; Guessennd et al. 2008). La présence des BMR dans les eaux de surface pourrait donc être un maillon important dans la dissémination et la circulation des gènes de résistance véhiculés par ces bactéries. L'objectif de cette étude était de détecter les BMR dans les effluents hospitaliers et d'évaluer leur niveau de résistance vis-à-vis de certains antibiotiques.

correspondant à trois prélèvements par jour sur cinq jours ont été effectués par semaine. Les BMR recherchées étaient les entérobactéries productrices de Bêta Lactamase à Spectre Elargie (EBLSE), Acinetobacter baumanii résistant à la ticarcilline (ABRT), Pseudomonas aeruginosa résistant à la ceftazidime (PARC), Staphylococcus aureus résistant à la méthicilline (SARM) et Enterococcus résistant à la vancomycine (ECRV). Le dénombrement de la flore totale et de la flore résistante (BMR) s'est effectuée respectivement sur la gélose Plate count Agar ${ }^{\circledR}$ (610040 Liofilchem) et sur des milieux sélectifs « faits 
maison » contenant des antibiotiques, après une série de dilution décimale. Les géloses ont été incubées à $37^{\circ} \mathrm{C}$ pendant 24 heures. La gélose Drigalski contenant $2 \mathrm{mg} / \mathrm{l}$ de ceftazidime, la gélose Cétrimide contenant $2 \mathrm{mg} / \mathrm{l}$ de ceftazidime, le milieu sélectif contenant $4 \mathrm{mg} / \mathrm{l}$ de tobramycine et la gélose Bile Esculine Agar (BEA) contenant $6 \mathrm{mg} / \mathrm{l}$ de vancomycine ont été utilisées pour l'isolement respectif des EBLSE, ABRT, PARC, SARM et des ECRV (Régnier, 1996 ; Bernet et Fines, 2000). Les mêmes milieux sans antibiotiques ont été utilisés comme preuve de viabilité des bactéries dans les prélèvements. Les souches de références utilisées comme témoins sont listés dans le tableau 1.

Tableau 1 : souches de référence utilisées pour l'évaluation des milieux

\begin{tabular}{l} 
Souches de référence \\
\hline Escherichia coli U2A 1528 (AAC 6') \\
Proteus mirabilis U2A 1878 (Cit/Fox) \\
Klebsiella pneumoniae U2A 2239 (Tem 1 ) \\
Escherichia coli ATCC 29922 \\
Staphylococcus sp 573/09 et 978/09 \\
Staphylococcus aureus ATCC 29213 \\
Pseudomonas Imp-1U2A2257 \\
Pseudomonas aeruginosa ATCC 27853 \\
Enterococcus U2A 432 \\
Enterococcus 620/09
\end{tabular}

Les galeries API 20E® de biomérieux (Marcy l'étoile, France) et API STAPH® de Biomérieux (Marcy l'étoile, France) ont été utilisés pour l'identification des souches. Un antibiogramme a été réalisé par la méthode de diffusion de disques selon les normes du CASFM 2011 en vue d'étudier la résistance vis-à-vis des autres familles d'antibiotiques. Les antibiotiques testés sont consignés dans le tableau 2. Pour la détection des EBLSE, les céphalosporines de 3ème génération (céftriaxone (CRO), céfépime (FEP), céfotaxime (CTX), ceftazidime (CAZ) et aztréonam

\section{Phénotypes}

BLSE $(+)$

$\operatorname{BLSE}(+)$

BLSE $(+)$

BLSE(-)

Méthicilline résistant

Méthicilline sensible

Ceftazidime résistant

Ceftazidime sensible

Vancomycine résistant

Vancomycine sensible

Tableau 2 : Liste des disques d'antibiotiques testés par genre bactérien et leurs break points selon le CA-SFM 2011.

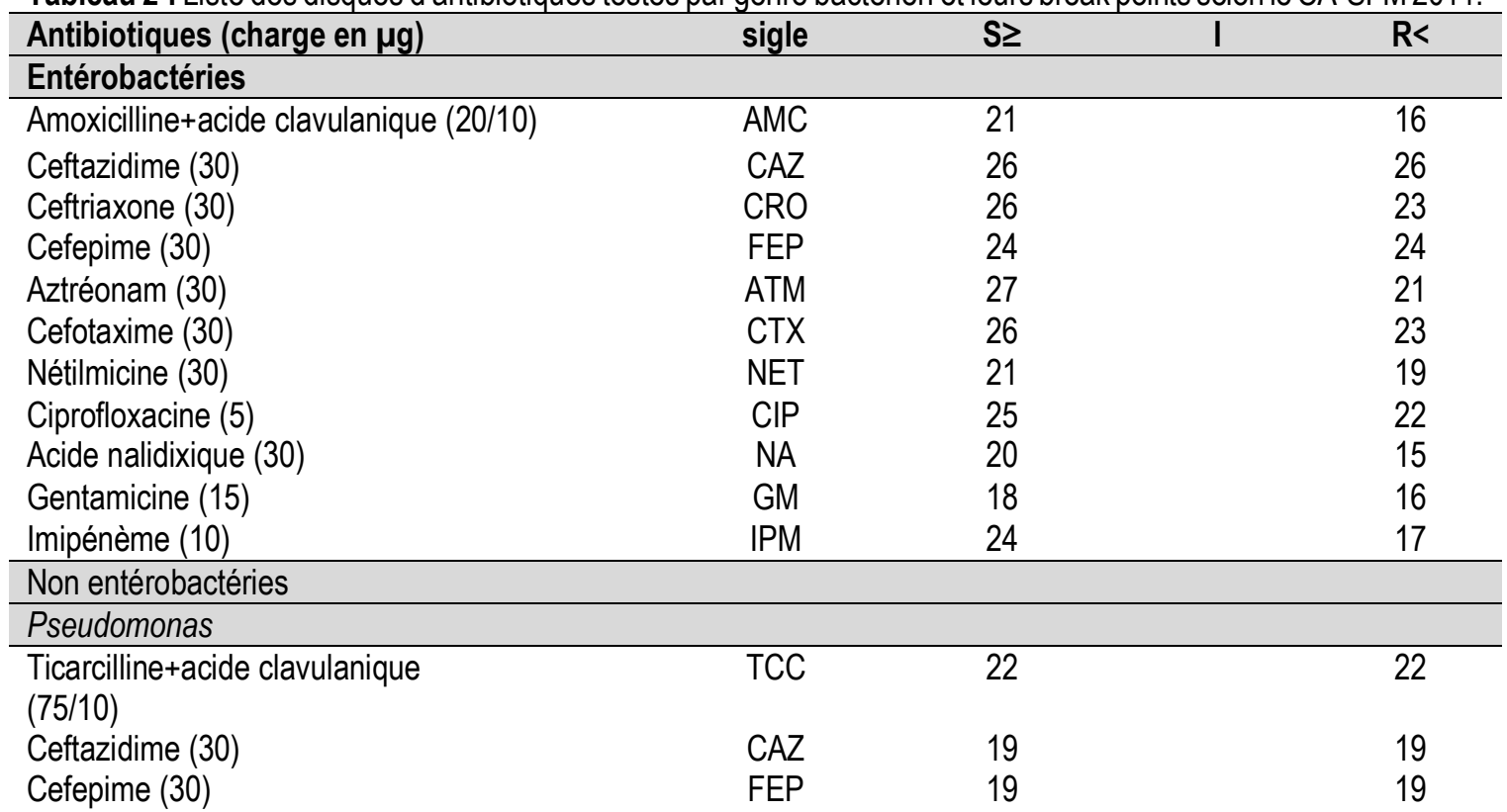

(ATM) ont été placés à $1,5 \mathrm{~cm}$ du disque de l'amoxicilline associé à l'acide clavulanique (AMC). La présence d'une image de synergie traduit la production d'une bêta-lactamase. Le contrôle de qualité des antibiogrammes a été effectué avec les souches de référence d'Escherichia coli ATCC 29922, Staphylococcus aureus ATCC 29213 et de Pseudomonas aeruginosa ATCC 27853. La liste des antibiotiques et leur charge testés est donnée dans le tableau 2 
Guessennd et al. J. Appl. Biosci. 2013. Étude des bacteries multiresistantes des effluents hospitaliers de la ville d, Abidjan

\begin{tabular}{lccc}
\hline Aztréonam (30) & ATM & 27 & 19 \\
Imipénème (10) & IPM & 22 & 17 \\
\hline Acinetobacter baumanii & & & 22 \\
\hline Ticarcilline (75) & TIC & 22 & 19 \\
Cefepime (30) & FEP & 19 & 17 \\
Imipénème (10) & IPM & 22 & 19 \\
Ciprofloxacine (5) & CIP & 22 & 16 \\
Gentamicine (15) & GM & 16 & 25 \\
\hline Staphylococcus spp & & & 20 \\
\hline Cefoxitine (30) & FOX & 27 & - \\
Oxacilline (5) & OX & 20 & 24 \\
Vancomycine (30) & VA & 17 & 21 \\
Acide fusidique (10) & FA & 24 & \\
Tétracycline (30) & TE & 23 & \\
\hline
\end{tabular}

\section{RÉSULTATS}

Dénombrement : Après le dénombrement de la flore totale bactérienne, une concentration moyenne en germe de $10,3410^{3} \mathrm{UFC} / \mathrm{ml}$ et une concentration moyenne de la flore résistante (BMR) de $90 \mathrm{UFC} / \mathrm{ml}$ a été obtenue. Selon la morphologie des bactéries, les

bacilles à Gram négatif étaient prédominants avec $82,4 \%$. Au total 136 bactéries ont été isolées et retenues pour les tests de sensibilité. La répartition des bactéries est donnée dans le tableau 3.

Tableau 3 : Fréquence des genres et espèces bactériens isolés.

\begin{tabular}{lcc}
\hline Souches & Nombre & $(\%)$ \\
\hline Entérobactéries & & \\
\hline Escherichia coli & 17 & 34,7 \\
Klebsiella pneumoniae & 16 & 32,7 \\
Klebsiella oxytoca & 1 & 2,0 \\
Enterobacter aerogenes & 11 & 22,4 \\
Enterobacter cloacae & 2 & 4,1 \\
Citrobacter koseri & 2 & 4,1 \\
\hline Sous-total & 49 & 100 \\
\hline Non entérobactéries & & \\
\hline Pseudomonas aeruginosa & 45 & 72,4 \\
Pseudomonas putida & 4 & 6,3 \\
Pseudomonas mendocina & 1 & 1,6 \\
Pseudomonas putrefaciens & 1 & 1,6 \\
Acinetobacter baumanii & 11 & 17,5 \\
Aeromonas & 1 & 1,6 \\
\hline Sous-total & 63 & 100 \\
\hline Cocci & & \\
\hline Staphylococcus spp & 24 & 100 \\
\hline Total & 136 & 100 \\
\hline
\end{tabular}

Les taux de résistance aux différents antibiotiques testés sur les principales souches isolées sont présentés dans le tableau 4. 
Tableau 4 : Taux de résistance aux antibiotiques des principales espèces isolées Antibiotiques

Nombre de souches résistantes (I+R \%)

\begin{tabular}{|c|c|c|c|c|c|c|}
\hline & $\begin{array}{l}\text { E. coli } \\
(\mathrm{N}=17)\end{array}$ & $\begin{array}{l}\text { K. } \\
\text { Pneumoniae }(\mathrm{N}=16)\end{array}$ & $\begin{array}{l}\text { E. } \\
\text { Aerogenes }(\mathrm{N}=11)\end{array}$ & $\begin{array}{l}\text { P. aeruginosa } \\
(\mathrm{N}=45)\end{array}$ & $\begin{array}{l}\text { Staphylococcus aureus } \\
(\mathrm{N}=24)\end{array}$ & $\begin{array}{l}\text { A.baumanii } \\
(\mathrm{N}=11)\end{array}$ \\
\hline Ticarcilline $75 \mu \mathrm{g}$ & - & - & - & - & - & $11(100)$ \\
\hline $\begin{array}{l}\text { Amoxicilline+acide clavulanique } \\
20 / 10 \mu \mathrm{g}\end{array}$ & $17(100)$ & $16(100)$ & $11(100)$ & - & - & - \\
\hline Cefotaxime $30 \mu \mathrm{g}$ & $17(100)$ & $15(93,8)$ & $11(100)$ & - & - & - \\
\hline Ceftriaxone $30 \mu \mathrm{g}$ & $17(100)$ & $16(100)$ & $11(100)$ & - & - & - \\
\hline Ceftazidime $30 \mu \mathrm{g}$ & $17(100)$ & $16(100)$ & $11(100)$ & $45(100)$ & - & $10(90,9)$ \\
\hline Cefepime $30 \mu \mathrm{g}$ & $17(100)$ & $14(87,5)$ & $11(100)$ & $45(100)$ & - & $8(72,7)$ \\
\hline Aztreonam $20 \mu \mathrm{g}$ & $17(100)$ & $16(100)$ & $11(100)$ & $37(82,2)$ & - & - \\
\hline Imipénème 10 g g & $0(0)$ & $0(0)$ & $0(0)$ & $36(80)$ & - & $0(0)$ \\
\hline $\begin{array}{l}\text { Ticarcilline+acide clavulanique } \\
75 / 10 \mu g\end{array}$ & - & - & - & $45(100)$ & - & $11(0)$ \\
\hline Acide nalidixique $30 \mu \mathrm{g}$ & $17(100)$ & $14(87,5)$ & $11(100)$ & - & - & - \\
\hline Ciprofloxacine $5 \mu \mathrm{g}$ & $17(100)$ & $10(62,5)$ & $10(90,9)$ & $36(80)$ & - & $3(27,7)$ \\
\hline Gentamicine $15 \mu \mathrm{g}$ & $13(76,5)$ & $50(50,0)$ & $7(63,6)$ & $38(84,4)$ & $24(100)$ & $7(63,6)$ \\
\hline Nétilmicine $30 \mu \mathrm{g}$ & $14(82,4)$ & $9(56,3)$ & $7(63,3)$ & - & - & $3(27,7)$ \\
\hline Oxacilline $5 \mu \mathrm{g}$ & - & - & - & - & $24(100)$ & - \\
\hline Cefoxitine $30 \mu \mathrm{g}$ & - & - & - & - & $24(100)$ & - \\
\hline Tétracycline $30 \mu \mathrm{g}$ & - & - & - & - & $24(100)$ & - \\
\hline Vancomycine $30 \mu \mathrm{g}$ & - & - & - & - & $12(50)$ & - \\
\hline Acide fusidique $10 \mu \mathrm{g}$ & - & - & - & - & $24(100)$ & - \\
\hline
\end{tabular}

(-) signifie non testé 
Tableau 5 : Répartition des phénotypes selon les principales espèces bactériennes Souches

Phénotype de résistance $\mathrm{N}(\%)$

\begin{tabular}{lc}
\hline Entérobactéries & NA CIP GM NET \\
\hline Escherichia coli & $13(29,4)$ \\
Klebsiella pneumoniae & $05(31,3)$ \\
Enterobacter aerogenes & $06(54,5)$ \\
\hline Non entérobactéries & TCC CAZ FEP ATM IPM \\
\hline Pseudomonas aeruginosa & $27(60,0)$ \\
& CAZ FEP CIP GM \\
Acinetobacter baumanii & $3(27,3)$ \\
\hline Cocci & FOX GM TE VA FA \\
\hline Staphylococcus aureus & $11(45,8)$ \\
\hline
\end{tabular}

\section{DISCUSSION}

La numération de la flore totale bactérienne donne une concentration moyenne en germe de 10,3.105 UFC/ml, inférieure à celle notée par Ekhaise et al en 2008 au cours de leurs travaux au Benin où la population bactérienne était comprise entre $1,9.10^{7} \mathrm{UFC} / \mathrm{ml}$ et $8,3.10^{12} \mathrm{UFC} / \mathrm{ml}$ et supérieure à celle trouvée par Bernet et Fines en 2000 où la concentration moyenne en germe était de $3.10^{5} \mathrm{UFC} / \mathrm{ml}$. Cette concentration moyenne en germe est inférieure à la flore totale généralement présente dans les eaux usées $\left(10^{8}\right.$ UFC/ml) (Bernet et Fines, 2000). La faible concentration pourrait s'expliquer par la présence probable de résidus de substances spécifiques dans les effluents (désinfectants, antiseptiques etc.) (Nunez et Moretton, 2007). La présence de résidus d'antibiotiques non métabolisés dans les effluents hospitaliers contribuerait largement à la sélection des bactéries multirésistantes dans les eaux usées (Islam et al., 2008).

Dans plusieurs études réalisées en Afrique (Benin, Maroc) et en Europe (Espagne, France), les auteurs ont également mise en évidence une flore bactérienne des les effluents hospitaliers dominée par les bactéries à Gram négatif appartenant à la famille des Enterobacteriaceae (E. coli, K. pneumoniae) et des Pseudomonaceae (Pseudomonas aeruginosa) (Marisol et al., 2000; Jeannette et al., 2007 ; Ekhaise, 2008; Atef et al., 2008) et dans une moindre mesure les Staphylocoques (Coralie et al., 2002). Cette flore mise en évidence était composée de la flore des malades et de l'environnement hospitaliers (sols, surfaces, matériels, eau, air).

Toutes les 49 souches d'entérobactéries isolées produisaient une bêta-lactamine à spectre élargi (BLSE) et étaient sensibles à l'imipenème. Sur l'ensemble des souches, $63,3 \%$ étaient résistantes à la fois à aux moins 3 familles d'antibiotiques (bêta- lactamines, aminosides et fluoroquinolones). L'observation de cette multi-résistance traduit bien la présence des BMR dans les effluents hospitaliers et à l'hôpital comme le montre les travaux de Guessennd et al en 2008 et de Dadié et al en 2003. Cela pourrait s'expliquer d'une part par la pression de sélection exercée par les praticiens et d'autres part par la présence de faibles concentrations d'antibiotiques non métabolisé rejetées par l'hôpital (Guardabassi et al., 1998, Jeannette et al., 2007 ; Thomas et al., 2007 ;). Ces résultats sont comparables à ceux d'Islam et al qui ont trouvés dans leurs travaux des bactéries multirésistantes à 5 familles d'antibiotiques avec un taux de résistance de $100 \%$ à la ciprofloxacine, à la tétracycline à la pénicilline et à l'érythromycine. Par contre les taux étaient de $50 \%$ et $90 \%$ respectivement pour la gentamycine et le chloramphénicol (Islam et al., 2008). Ces différents phénotypes de multi-résistance pourraient s'expliquer aussi par le fait que les antibiotiques font partie des molécules les plus prescrites en Afrique et parmi ces antibiotiques les bêta-lactamines venaient en tête (Dosso et al., 2000). Selon Philippon et al, (Philippon et Lagrange, 1994), les bactéries productrices de BLSE, de par leur déterminisme génétique sont souvent résistantes à plusieurs autres antibiotiques.

Sur l'ensemble des Pseudomonas aeruginosa, 58,8 \% présentaient une résistance à la ticarcilline, à l'association ticarcilline-acide clavulanique, à la ceftazime, à la cefepime, à l'aztreonam et à l'imipenème comme certaines souches de l'environnement hospitalier (Gbonon et al., 2007). Ces résistantes observées aux niveaux des Pseudomonas aeruginosa pourraient suggérer une céphalosporinase de haut niveau et même une carbapénèmase comme les souches hospitalières (Dadié et al., 2003). Pseudomonas aeruginosa est caractérisé par son 
niveau élévé de résistance naturelle et par son aptitude à acquérir et cumuler de nombreux et variés mécanismes de résistances aussi bien en milieu hospitalier que dans l'environnement sous l'effet de la sélection exercée par les antibiotiques : sécrétion de bêtalactamases, modification de la perméabilité membranaire (efflux, imperméabilité) et modification de cible, notamment des topoisomérases (Bert et al., 1999; Defez et al., 2004; Mesaros et al., 2007). La résistance des $P$ seudomonas aeruginosa à la fois aux bêtalactamines, aux aminosides et aux fluoroquinolones pourrait compromettre les options de traitement en cas d'infection à ces Pseudomonas aeruginosa. D'une manière générale, l'initiation du traitement d'une infection Pseudomonas aeruginosa repose toujours sur une association de deux antibiotiques pour prévenir l'émergence de mutants résistants. Les associations fréquemment utilisées sont les bêtalactamines et les fluoroquinolones, les bêtalactamines et les aminosides ou les aminosides et les fluoroquinolones (Mesaros et al., 2007).

Toutes les souches de Staphylococcus étaient des méticillines résistants et résistants à la gentamycine donc de phénotype kanamycine, tobramycine, gentamycine (KTG), ces souches sont reconnues comme ayant une grande capacité d'acquisition de gènes de résistance.-(Teresa et al., 1984). Parmi les

\section{CONCLUSION}

II ressort de cette étude que les effluents du Centre Hospitalier et Universitaire (CHU) de Cocody renferme la plupart des bactéries multi-résistantes impliquées dans les infections nosocomiales à savoir les entérobactéries productrices de Béta-Lactamines à Spectre Elargi (BLSE), les Acinetobacter baumanii multirésistants, les Pseudomonas résistants à la ceftazidime (PARC), les Staphylococcus aureus résistant à la méthicilline (SARM). Ces bactéries

\section{RÉFÉRENCES BIBLIOGRAPHIQUES}

Akoua Koffi C., Guessennd N., Gbonon V., Faye Ketté H., Dosso M., 2004. Methicillin resistance of Staphylococcus in Abidjan 1998-2001: A new problem. Medecine et maladies infectieuses; 34(3):132-6.

Atef M. Diab, Idriss M. Al-turk, Mohames K. Ibrahim, Khalil D. Al-Zhrany., 2008. Tracing of Gramnegative antibiotic-resistant bacteria in hospitals final affluent at Al-Mounwwarah. Journal of Taibah University for Science (JTUSCI) 1:23-34.

Berche P, Gaillard JL, Simonet M. In Bactériologie. Paris : Flammarion : 1988. P. 267-275. aminosides, la plus active était la gentamycine. Ces résultats sont supérieurs à ceux d'Akoua et al en 2004 où la proportion des SARM et de phénotype KTG étaient de $77,6 \%$ et $5,9 \%$ à la vancomycine en milieu hospitalier (Le coutumier et al., 1996; Akoua et al.,2004). La résistance des SARM à de nombreux antibiotiques a été aussi observée dans des études avec le taux de résistance de $90 \%$ à $95 \%$ pour les aminosides et de $90 \%$ pour les fluroquinolones (Berche et al., 1998, Ekhaise et Omavwoya, 2008). La présence de souche résistant à la vancomycine (très rare en milieu hospitalier pourrait être du à l'acquisition de plasmide de résistance par les Staphylococcus à travers un transfert horizontal entre les souches d'entérocoques de l'environnement qui ont une résistance naturelle à la vancomycine. Le risque sanitaire que font craindre la présence des Staphylococcus résistants à la vancomycine est le transfert de ces gènes de résistances aux souches hospitalières. Toutes les souches d'Acinetobacter isolées produisent une bêta-lactamine à spectre élargi (BLSE) synonyme d'une résistance aux bêtalactamines avec $27,3 \%$ de résistance à la ciprofloxacine et la gentamycine. Ces résultats ont été observés aussi par Ekhaise et Omavwoya en 2008 au cours de leurs travaux. Cette bactérie se distingue par son caractère multirésistant (Berche et al, 1998).

multirésistantes présentes et résistantes à deux ou trois familles d'antibiotiques peuvent causer des problèmes de santé publique. Ces effluents rejetés sans traitement préalable dans la lagune peuvent constituer une source de dissémination de ces bactéries potentiellement pathogènes. La mise en place de station de traitement par les autorités est primordiale afin réduire le risque sanitaire de ces effluents hospitaliers.

Bert F., Lambert-Zechovsky N., 1999 Résistance aux antibiotiques et problèmes thérapeutiques posés par Pseudomonas aeruginosa. Presse Med $28: 451-8$.

Coralie Darsy, Irène Lescure, Véronique Payot, Géraldine Rouland., 2002. Effluents des établissements hospitaliers et la maîtrise de la diffusion des bactéries multi résistantes: teneur en microorganismes pathogènes, risques sanitaires, procédures particulières d'épuration et gestion des boues, 10p.

Dadie A. T., Guessennd N., Tiekoura B., Faye-Kette H., Dosso M., 2003. Résistance aux bêtalactamines d'Escherichia coli d'origine 
alimentaire et humaine isolés à Abidjan. $\mathrm{J}$. Sci. Pharma. Biol. 4 (1) :62-69.

Defez C., Fabbro-Peray P., Bouziges N., Gouby A., Mahamat A., Daurès J. P., 2004. Risk factors for multidrug-resistant Pseudomonas aeruginosa nosocomial infection. J Hosp Infect ; 57:209-16.

Dosso M, Bissagnene E, Coulibaly M., 2000. Résistances Acquises Et Prescriptions d'antibiotiques en Afriques: quelles adéquations ? Med Mal Infec ; $30: 197-204$.

Ekhaise F.O., Omavwoya B.P., 2008. Influence of wastewater discharged from university of Benin reaching Hospital (UBTH), Benin City on its receiving environment. AmericanEurasian J. Agric and Environ. Sci., 4 (4): 484488.

El Rhazi K., S. Elfakir, M. Berraho N. Tachfouti, Z. Serhier, C. Kanjaa et C Nejjari. 2007. Prévalence et facteurs de risque des infections nosocomiales au CHU Hassan II de Fès (Maroc). Heath Journal volume 13 No. 1 January-February.

Gbonon V. C., Guessennd K. N., Kouassi M'bengue A., Kacou N'douba A., N'guessan Kouassi Raymond, Faye Kette H., Dosso M., Mignonsin D., 2007. Contrôles bactériologiques de l'environnement des blocs opératoires dans un pays en développement : cas du CHU de Treichville à Abidjan en l'an 2000. Revue Bio-Africa N 4 , pp. 7-11.

Guardabassi L., Petersen A., Olsen J. E and Dalsgaard A., 1998. Antibiotic resistance in Acinetobacter spp. Isoled from sewers receiving waste effluent from a hospital and a pharmaceutical plant. Appl. Environ Microbiol. 64 (9) : 34993502.

Guessennd K. N. ; Loubienga S. W. ; Gbonon V. ; Kouassi M'Bengue A. ; Kacou N'Douba A. ; Dosso M. 2004. Résistance aux antibiotiques de 241 souches de Escherichia coli isolées des infections urinaires des patients hospitalisés au $\mathrm{CHU}$ de Cocody à Abidjan. J. Sci. Biol., Vol. 5, n¹- pp. 38-45.

Guessennd N., V. Gbonon, A. Kacou N'douba, S. W. Loubienga, H. Faye-Kette, M. Dosso. Niveau de prévalence aux antibiotiques de 351 souches de Pseudomonas aeruginosa isolées d'infections pariétales post opératoires à Abidjan de 1998 à 2003, 2005. J. Sci. Pharm. Biol., vol $6 \mathrm{n}^{\circ} 2$, pp 66-72.

Guessennd N.; S. Bremont; V. Gbonon; A. KacouN'Douba; E. Ekaza; T. Lambert; M. Dosso; P. Courvalin., 2008. Résistance aux quinolones de type qnr chez lez entérobactéries productrices de bêta-lactamases à spectre élargi à Abidjan en Côte d'Ivoire. Pathologie Biologie 56 : 439-446.

Islam M. J., M. S. Uddin, M. A. Hakim, K. K. Das, M. N. Hasan. Role of untreated liquid hospital waste to the development of antibiotic resistant bacteria. 2008. J. Innov. Dev. Strategy.2 (2):17-21.

Jeannette Munez-Aguayo, Kelvin S. Lang, Timothy M Lapara Gerald Gonzàlez and Randall S. Singer., 2007. Evaluating the Effect of Chlortetracycline on the proliferation of Antibiotic-resistant bacteria in a stimuled river water ecosystem. Applied and Environnemental Microbiology. 73 (7): 5421 5425.

Le Coutumier A, Geudet P, Lecaillon E., 1996. Staphylococcus aureus méthicillino-résistant : Passé présent, futur $1^{\text {re }}$ et $2^{\mathrm{e}}$ partie. Spectra Biol ; 79 :-7.

Marisol G, Capdepuy M., Arpin C., Raymond N., Caumette P., and Quentin C., 2000. Impact of an urban effluent on antibiotic resistance of riverine enterobacteriaceae and aeromonas spp. Applied and Environmental Microbiology. 66(1): 125-132.

Mehmet Faruk Geyik, Salih Hosoglu, Celal Ayaz, Mustafa Kemal Celen, Cemal Ustun, 2008. Surveillance of nosocomial infections in Dicle University Hospital: a ten year Experience. Turk J Med; 38 (6): 587-593.

Mesaros N., Nordmann P., Plésiat P., RousselDelvallez M., Van Eldere J., Glupczynnski Y., 2007. Pseudomonas aeruginosa: resistance and therapeutic options at the new millennium. Clin Micribiol Infect; 13:560-78

Nuñez L.; J. Moretton., 2007. Disinfectant- Resistant bacteria in Buenos aires city hospital waste water. Brazilian. Journal of Microbiology 38:644-648.

Philippon A, Arlet G, and Lagrange P.H., 1994. Origin and impact of plasmid-mediated extendedspectrum beta lactamases Eur J Clin Microbial Infec Dis; 13 Suppl 1:S17-29.

Régnier R., 1996. Les bactéries multirésistantes aux antibiotiques en réanimation : contexte épidémiologique et stratégies de maîtrise. Path Biol ; 44, Spécial:113-123.

Senka Dzidic, Vladimir Bedekovic., 2003. Horizontal gene transfer-emergency multidrug resistance in hospital bacteria. Acta Pharmacol; 24 (6): $519-526$ 
Sophie Bernet, Marguerite Fines. Effluents du CHU de CAEN : Etude quantitative et qualitative de la flore microbienne et recherche de bactéries multi résistantes (2000). Quatrième journée du Réseau Régional d'Hygiène de BasseNormandie, Caen (communication affichée).

Teresa C. Horan, John W. White, William R. Jarvis 1984. Nosocomial infection surveillance. Surveillance summaries December 01, 1986/ 35(SS-1); 17-29.
Thomas S., Holger V., Slike K., Wolfagang K., Katja S., Bernd J. and Ursula O., 2007. Detection of antibiotic- resistant bacteria and their resistance genes in waster, surface water and drinking water biofilms, FEMS Microbiology Ecology. 43 (3):325-335. 